\title{
RECURSIVE EMBEDDINGS OF PARTIAL ORDERINGS
}

\author{
K. R. APT
}

Introduction. Let $\mathscr{A}$ be a countable atomless Boolean algebra and let $X$ be a countable partial ordering. We prove that there exists an embedding of $X$ into $\mathscr{A}$ which is recursive in $X, \mathscr{A}$ and which destroys all suprema and infima of $X$ which can be destroyed. We show that the above theorem is false when we try to preserve all suprema and infima of $X$ instead of destroying them. Finally we indicate that if $\mathscr{A}$ and $\mathscr{B}$ are countable Boolean algebras and $\mathscr{B}$ is atomless then $\mathscr{A}$ can be embedded into $\mathscr{B}$ by a function which is recursive in $\mathscr{A}, \mathscr{B}$. If $\mathscr{A}$ is also atomless, then there is an isomorphism from $\mathscr{A}$ into $\mathscr{B}$ which is recursive in $\mathscr{A}, \mathscr{B}$.

1. Preliminaries. Throughout the paper $\omega$ denotes the set of natural numbers, and $\phi$ the empty set. If $X$ is a set and $n$ a natural number then $X^{n}$ denotes the set of all $n$-tuples of elements of $X$. We say that $X$ is a partial ordering on a set $A$ (p.o. on $A$ ) if for some $B \subset A X \subset B^{2}$ and for all $x, y$, $z \in B$

1) $(x, x) \in X$,

2) $((x, y) \in X \wedge(y, x) \in X) \Rightarrow x=y$,

3) $((x, y) \in X \wedge(y, z) \in X) \Rightarrow(x, z) \in X$.

If $(x, y) \in X$, we write $x \leqq_{X} y$. If $(x, y) \in X \wedge x \neq y$, we write $x<_{x} y$. If $(x, y) \notin X$ and $(y, x) \notin X$, we say that $x$ and $y$ are $X$-incomparable and we write $x \|_{x} y$.

$z$ is called the supremum of $x$ and $y$ in $X(x \cup y=z)$, if

$$
x \leqq_{X} z \wedge y \leqq_{X} z \wedge \forall t\left[\left(x \leqq_{X} t \wedge y \leqq_{X} t\right) \Rightarrow_{z} \leqq_{X} t\right],
$$

and $z$ is called the infinum of $x$ and $y$ in $X(x \cap y=z)$, if

$$
z \leqq_{X} x \wedge z \leqq_{X} y \wedge \forall t\left[\left(t \leqq_{x} x \wedge t \leqq_{x} y\right) \Rightarrow_{t} \leqq_{x} z\right] .
$$

By Fld $(X)$ we denote the set $\{x:(x, x) \in X\}$.

For the definition of a Boolean algebra we refer the reader to Sikorski [4]. If $\mathscr{A}$ is a Boolean algebra then 0 denotes its smallest element and 1 the greatest one. If $x$ and $y$ are elements of $\mathscr{A}$, then we write $x \leqq y$ if $x \cup y=y$ and $x<y$ if $x \leqq y$ and $x \neq y$. We write $x \| y$ if $\urcorner(x \leqq y)$ and $\urcorner(y \leqq x)$.

Received April 29, 1976. This paper is registered as Report ZW 67/76 of the Mathematical Centre. Some of the results of this paper were obtained in 1971 when the author was a student at Wrocław University, Poland. 
We say that $\mathscr{A}$ is a Boolean algebra on a set $A$, if every element of $\mathscr{A}$ is an element of $A$.

In this paper we are interested in partial orderings on $\omega$ and Boolean algebras on $\omega$.

Definition 1. Let $X$ be a p.o. on a set $A$ and $\mathscr{A}$ a Boolean algebra. $f$ is called an embedding of $X$ into $\mathscr{A}$ if $f$ is an injective function from Fld $(X)$ into $\mathscr{A}$ such that for all $x, y \in$ Fld $(X)$

$$
x<_{x} y \Leftrightarrow f(x)<f(y) .
$$

We say that an embedding $f$ of $X$ into $\mathscr{A}$ preserves all suprema and infima of $X$ if

I) whenever $x \cup y=z$, then $f(x) \cup f(y)=f(z)$; and

II) whenever $x \cap y=z$, then $f(x) \cap f(y)=f(z)$.

We say that an embedding $f$ of $X$ into $\mathscr{A}$ destroys all suprema and infima of $X$ if

I) whenever $x \|_{x} y$ and $x \cup y=z$, then $f(x) \cup f(y) \neq f(z)$; and

II) whenever $x \|_{x} y$ and $x \cap y=z$, then $f(x) \cap f(y) \neq f(z)$.

Observe that if $x \leqq x y$, then $x \cup y=y$ and $x \cap y=x$, so for any embedding $f$ of $X$ into $\mathscr{A}, f(x) \cup f(y)=f(x \cup y)$ and $f(x) \cap f(y)=f(x \cap y)$. Thus an embedding of $X$ in to $\mathscr{A}$ cannot destroy suprema and infima of $X$-comparable elements.

All the notions from recursion theory we use can be found in Shoenfield [2]. In particular, $\mathrm{Seq}(x)$ means that $x$ codes a finite sequence of natural numbers, and $\operatorname{lh}(x)$ is the length of that sequence. If Seq $(x)$ then $x=\left\langle(x)_{0}, \ldots,(x)_{\ln (x)-1}\right\rangle$. If $a=\left\langle a_{1}, \ldots, a_{n}\right\rangle$ and $b=\left\langle b_{1}, \ldots, b_{n}\right\rangle$, then $a * b=\left\langle a_{1}, \ldots, a_{n}, b_{1}, \ldots, b_{n}\right\rangle$. All the mentioned functions and relations are recursive.

If $A=\left\{a_{1}, \ldots, a_{k}\right\}$ then $x$ is called the code of $A(x=\langle A\rangle)$ if $x$ is the least number $z$ such that seq $(z), \operatorname{lh}(z)=k$ and $\left\{(z)_{i}: i<\operatorname{lh}(z)\right\}=A$. If $f\left(x_{1}, \ldots, x_{n}\right)$ is a function then $\operatorname{graph}(f)=\left\{\left(x_{1}, \ldots, x_{n}, y\right): f\left(x_{1}, \ldots, x_{n}\right)=y\right\}$.

Definition 2. Let $\mathscr{A}=\langle A, \cup, \cap,-, 0,1\rangle$ be a Boolean algebra on $\omega$. We say that $f$ is recursive in $\mathscr{A}$ if $f$ is recursive in $\{A, \operatorname{graph}(\cup), \operatorname{graph}(\cap)$, $\operatorname{graph}(-)\}$.

Similarly we say that $f$ is recursive in $\mathscr{A}, \mathscr{B}$ where $\mathscr{B}$ is another Boolean algebra on $\omega$ or that $f$ is recursive in $X, \mathscr{A}$ for a set $X$.

Definition 3. Let $\mathscr{A}$ be a Boolean algebra. Suppose that $A$ and $B$ are sets of elements of $\mathscr{A}$. Then

I) if $a \leqq b$ for all $a \in A, b \in B$ we write $A \leqq B$;

II) if $a<b$ for all $a \in A, b \in B$ we write $A<B$;

III) if $\urcorner(a \leqq b)$ for all $a \in A, b \in B$ we write $A \$ B$;

IV) if $a \| b$ for all $a \in A, b \in B$ we write $A \| B$.

Instead of $\{a\}<A$ we write $a<A$. Similarly with other relations. Observe that for every set $A, \phi<A, A<\phi, \phi \supsetneqq A, A \nsubseteq \phi$ and $\phi \| A$. 
If $A$ is a finite set of elements of $\mathscr{A}$ then sup $A$ denotes the least element $a$ of $\mathscr{A}$ such that $A \leqq a$, and $\inf A$ denotes the greatest element $a$ of $\mathscr{A}$ such that $a \leqq A$. Observe that $\sup \phi=0$ and inf $\phi=1$. Recall that a Boolean algebra $\mathscr{A}$ is atomless if $0<x$ implies for some $y, 0<y<x$.

2. Embeddings destroying suprema and infima. In this section we prove the following theorem:

Theorem 1. Let $X$ be a partial ordering on $\omega$ and let $\mathscr{A}$ be an atomless Boolean algebra on $\omega$. Then there exists an embedding $f$ of $X$ into $\mathscr{A}$ such that

I) $f$ destroys all suprema and infima of $X$, and

II) $f$ is recursive in $X, \mathscr{A}$.

We first present an informal idea of the proof. Let Fld $(X)=\left\{a_{0}, a_{1}, \ldots\right\}$ be a recursive in $X$ enumeration of Fld $(X)$. We want to build the required embedding by induction. Suppose that for $i \leqq n$ we already defined some elements $b_{i}$ of $\mathscr{A}$ such that

$$
a_{i}<_{X} a_{j} \Leftrightarrow b_{i}<b_{j} \text { for } i, j \leqq n .
$$

We want to define an element $b_{n+1}$ of $\mathscr{A}$ such that

$\left(^{*}\right) \quad a_{i}<_{X} a_{j} \Leftrightarrow b_{i}<b_{j} \quad$ for $i, j \leqq n+1$.

If we do not impose any conditions on $b_{i}-s$ we can be stuck. For example, if $a_{0}<a_{2}, a_{1}<a_{2}, a_{0}<a_{3}, a_{1}<a_{3}$ and $a_{3}<a_{2}$ (represented schematically by the following diagram)

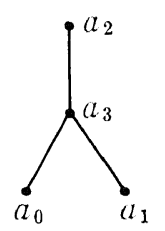

and we choose $b_{0}, b_{1}$ and $b_{2}$ in such a way that $b_{0} \cup b_{1}=b_{2}$ then there is no $b_{3}$ such that $b_{3}<b_{2}, b_{0}<b_{3}$ and $b_{1}<b_{3}$.

In order to prevent such situations we choose $b_{i}-s$ in a more careful way. For example, the above difficulty would not occur if $b_{0} \cup b_{1}<b_{2}$. Thus we assume that the elements $b_{0}, \ldots, b_{n}$ satisfy an additional property, namely that the set $\left\{b_{0}, \ldots, b_{n}\right\}$ is normal (see Definition 4 ).

Let

$$
\begin{aligned}
& A=\left\{b_{i}: a_{i}<_{X} a_{n+1}, i \leqq n\right\}, \\
& B=\left\{b_{i}: a_{n+1}<_{X} a_{i}, i \leqq n\right\}, \\
& C=\left\{b_{i}: a_{n+1} \|_{X} a_{i}, i \leqq n\right\} .
\end{aligned}
$$

Then $A \cup B \cup C=\left\{b_{0}, \ldots, b_{n}\right\}$. Observe that $A<B, C \neq A$ and $B \neq C$. Since $A \cup B \cup C$ is a normal set we get from this that $\sup A<\inf B, C$ 
$\sup A$ and inf $B \neq C$. We are looking for an element $b_{n+1}$ such that sup $A<$ $b_{n+1}<\inf B$ and $b_{n+1} \| C$. Then (*) holds. The existence of such a $b_{n+1}$ is guaranteed by Lemma 1.

But we want also to preserve our additional condition, so we claim also that the set $A \cup B \cup C \cup\left\{b_{n+1}\right\}$ is normal. Lemma 2 shows that the required $b_{n+1}$ still can be found. Its proof uses Lemma 1, but in an appropriately modified way.

Thus the induction step works. The obtained embedding destroys all suprema and infima of $X$ which is an immediate consequence of the fact that for each $n$, the set $\left\{b_{0}, \ldots, b_{n}\right\}$ is normal.

Choosing at each time the smallest $b_{n+1}$ satisfying the above conditions (see the definition of the function $g$ in the proof of Theorem 1 ) we ensure that the above embedding is recursive in $X, \mathscr{A}$.

We present now the precise proof of the theorem. We first prove two lemmata.

Lemma 1. Let $\mathscr{A}$ be an atomless Boolean algebra. Suppose that $A \cup\{a, b\}$ is a finite set of elements of $\mathscr{A}$, such that:

1) $a<b$;

2) $A \neq a$;

3) $b \$ A$.

Then there exists an element $c$ of $\mathscr{A}$, such that $a<c<b$ and $c \| A$.

Obviously Conditions 2) and 3) have to be satisfied if we want to prove the claim. The lemma shows that 2) and 3) are also sufficient conditions.

Proof. At first we "modify" $A$ to a set $A^{\prime}$ such that $a<A^{\prime}<b$. We find then an element $c$ such that $a<c<b$ and $c \| A^{\prime}$. It turns out that also $c \| A$.
Let

$$
A^{\prime}=\{b \cap d: d \in A \text { and } a<b \cap d\} \cup\{a \cup d: d \in A \text { and } a \cup d<b\} .
$$

Suppose that $x=b \cap d$ for some $d \in A$ such that $a<b \cap d$. Then $x \leqq b$. If $x=b$ then $b \leqq d$, which violates our assumptions. Thus $a<x<b$.

Suppose now that $x=a \cup d$ for some $d \in A$ such that $a \cup d<b$. Then $a \leqq x$. If $a=x$ then $d \leqq a$, which violates our assumptions. Thus $a<x<b$. So $a<A^{\prime}<b$.

We can treat the set $B=\{x: a \leqq x \leqq b\}$ as a Boolean algebra with the operations induced by $\mathscr{A}$.

$$
\begin{aligned}
x \dot{\cup} y & =x \cup y \\
x \dot{\cap} y & =x \cap y \\
\dot{0} & =a \\
\dot{1} & =b \\
-x & =a \cup(b \cap-x)
\end{aligned}
$$


Let $A^{\prime}=\left\{a_{1}, \ldots, a_{n}\right\}$. We just proved that $\dot{0}<a_{i}$ and $\dot{0}<-a_{i}$ for all $i \leqq n$. Let $C=\left\{b_{1} \cap \ldots \cap b_{n}\right.$ : for all $i \leqq n, b_{i}=a_{i}$ or $\left.b_{i}=-a_{i}\right\}$. Then each $a_{i}$ or $-a_{i}$ is a sum of elements of $C$. For each $i \leqq 2 n$, pick an element $c_{i}$ from $C$ such that

$$
\dot{0}<c_{j} \leqq a_{j} \text { and } \dot{0}<c_{j+n} \leqq-a_{j} \text { for all } j \leqq n .
$$

$\mathscr{A}$ is atomless so there exist elements $d_{i}$ such that for $i \leqq 2 n, \dot{0}<d_{i}<c_{i}$. We can choose $d_{i}-s$ in such a way that $d_{i}=d_{j}$ if $c_{i}=c_{j}$.

Finally let $c=d_{1} \cup \ldots \cup d_{2 n}$. We claim that $c$ is the desired element. We prove at first that $c \| A^{\prime}$. Suppose that for some $i \leqq n, c \leqq a_{i}$. Then

$$
\dot{0}<d_{i+n} \leqq a_{i} \text { and } d_{i+n}<-a_{i}
$$

which is clearly impossible. If for some $i \leqq n, a_{i} \leqq c$ then

$$
c_{i} \cap \dot{-} d_{i} \leqq a_{i} \leqq c .
$$

Observe that for $x, y \in C$ either $x=y$ or $x \cap y=\dot{0}$. Hence for $k \leqq n$ either $c_{k}=c_{i}$ or $c_{k} \cap c_{i}=\dot{0}$. In the first case $d_{k}=d_{i}$, in the second $d_{k} \cap\left(c_{i} \cap-d_{i}\right)$ $=\dot{0}$. So in both cases we obtain $d_{k} \cap\left(c_{i} \cap \dot{-} d_{i}\right)=\dot{0}$. Finally we obtain:

$$
c_{i} \cap \dot{-} d_{i}=c_{i} \cap \dot{-} d_{i} \cap c=\bigcup_{k=1}^{2 n} d_{k} \cap\left(c_{i} \cap \dot{-} d_{i}\right)=\dot{0}
$$

which contradicts the choice of $d_{i}$.

Observe that by construction $a<c<b$. We prove now that $c \| A$. Suppose that $x \in A$. There are 3 possible cases:

I) $x \| a$ and $x \| b$. Then for every $y$ such that $a \leqq y \leqq b, x \| y$, so in particular $x \| c$.

II) $x<b$. There are two possible cases:

1) $a \cup x<b$. Then $a \cup x \in A^{\prime}$. So $a \cup x \| c$. If $x \leqq c$ then $a \cup x \leqq c$, which is impossible; if $c \leqq x$ then $c \leqq \imath \cup x$, which is impossible. Thus $c \| x$.

2) $a \cup x=b$. If $x \leqq c$, then $a \cup x \leqq c$, so $b \leqq c$ which is impossible; if $c \leqq x$, then $a \leqq x$, so $a \cup x=x$, i.e. $b=x$ which contradicts our assumptions. Thus $c \| x$.

III) $a<x$. There are two possible cases:

1) $a<b \cap x$. Then $b \cap x \in A^{\prime}$, so $b \cap x \| c$. If $x \leqq c$, then $b \cap x \leqq c$, which is impossible. If $c \leqq x$, then $c \leqq b \cap x$, which is impossible. Thus $x \| c$.

2) $a=b \cap x$. If $x \leqq c$, then $x \leqq b$, so $b \cap x=x$, i.e. $a=x$ which contradicts our assumptions. If $c \leqq x$, then $c \leqq b \cap x$, i.e. $c \leqq a$ which is impossible. Thus $c \| x$.

This concludes the proof of the lemma.

Definition 4. Let $\mathscr{A}$ be a Boolean algebra. A finite set $T$ of elements of $\mathscr{A}$ is called normal if for all $A$ and $B$ such that $A \cup B \subset T$ we have

$A<B$ implies $\sup A<\inf B$, and

$A \neq B$ implies inf $A \neq \sup B$. 
Lemma 2. Let $\mathscr{A}$ be an atomless Boolean algebra. Suppose that for some finite sets $A, B$ and $C$ of elements of $\mathscr{A}$,

$A<B, C \$ A, B \nsubseteq C$, and $A \cup B \cup C$ is normal.

Then there exists an element a of $\mathscr{A}$ such that

$\sup A<a<\inf B, a \| C$, and $A \cup B \cup C \cup\{a\}$ is normal.

Proof. Let $S$ be a subalgebra of $\mathscr{A}$ generated by the set $A \cup B \cup C$. Let

$$
T=\{x: x \in S \wedge\urcorner(x \leqq \sup A) \wedge\urcorner(\inf B \leqq x)\} .
$$

The set $T$ is of course finite.

Since $A \cup B \cup C$ is normal we get from our assumptions and Lemma 1 that for some $a$ in $A$, sup $A<a<\inf B$ and $a \| T$. We claim that $a$ is the required element. If $c \in C$, then $c \neq A$ and $B \neq c$. Since $A \cup B \cup C$ is normal, $c \neq \sup A$ and $\inf B \neq c$. Thus $C \subseteq T$, i.e. $a \| C$.

It remains to prove that $A \cup B \cup C \cup\{a\}$ is normal. Let $K \cup L \subset A \cup$ $B \cup C$. We have to consider the following four possible cases:

1) $K<L$ and $a<L$. We prove then that $\sup (K \cup\{a\})<\inf L$. We always have $\sup (K \cup\{a\}) \leqq \inf L$ so suppose that $\sup (K \cup\{a\})=\inf L$. Then $\sup K \cup a=\inf L$, so $\inf L \cap-\sup K \leqq a$, which indicates that inf $L$ $\cap-\sup K \notin T$. There are two possibilities:

I) $\inf B \leqq \inf L \cap \sup K$. Then inf $B \leqq a$ which contradicts the choice of $a$.

II) $\inf L \cap-\sup K \leqq \sup A$. Then $\inf L \leqq \sup A \cup \sup K$, i.e. $\inf L \leqq$ $\sup (A \cup K)$. The assumption $a<L$ implies, by the choice of $a$, that $L \subset B$. Thus $A<L$ since $A<B$. So $A \cup K<L$. But $A \cup B \cup C$ is normal, so we get that $\sup (A \cup K)<\inf L$, which contradicts our previous statement.

2) $K<L$ and $K<a$. We prove that $\sup K<\inf (L \cup\{a\})$. We always have $\sup K \leqq \inf (L \cup\{a\})$, so suppose that $\sup K=\inf (L \cup\{a\})$. Then $\sup K=\inf L \cap a$, so $a \leqq \sup K \cup-\inf L$. This indicates that sup $K \cup$ $-\inf L \notin T$. There are two possibilities:

I) $\sup K \cup-\inf L \leqq \sup A$. Then $a \leqq \sup A$ which is impossible.

II) $\inf B \leqq \sup K \cup-\inf L$. Then $\inf B \cap \inf L \leqq \sup K$, i.e. inf $(B \cup L)$ $\leqq \sup K$. But $K<a$, so $K \subset A$, i.e. $K<B$. Thus $K<L \cup B$. Since $A \cup B$ $\cup C$ is normal we get sup $K<\inf (B \cup L)$, which contradicts the former statement.

3) $K \neq L$ and $a \neq L$. We prove that inf $(K \cup\{a\}) \$ L$. Suppose that $\inf (K \cup\{a\}) \leqq \sup L$, i.e. $\inf K \cap a \leqq \sup L$. Then $a \leqq \sup L \cup-\inf K$, so $L \cup-\inf K \notin T$. There are two possibilities:

I) $\sup L \cup-\inf K \leqq \sup A$. Then $a \leqq \sup A$, which contradicts the choice of $a$.

II) $\inf B \leqq \sup L \cup-\inf K$. Then $\inf B \cap \inf K \leqq \sup L$, i.e. inf $(B \cup K)$ $\leqq \sup L$. But $a \neq L$, so by the choice of $a, B \neq L$, i.e. $B \cup K \neq L$. Since $A \cup B \cup C$ is normal we get that $\inf (B \cup K) \neq$ sup $L$, which contradicts the former statement. 
4) $K \neq L, K \neq a$. We prove that inf $K$ sup $(L \cup\{a\})$. Suppose that inf $K \leqq \sup (L \cup\{a\})$, i.e. $\inf K \leqq \sup L \cup a$. Then $\inf K \cap-\sup L \leqq a$, so inf $K \cap-\sup L \notin T$. There are two possibilities:

I) $\inf K \cap-\sup L \leqq \sup A$. Then $\inf K \leqq \sup A \cup \sup L$, i.e. $\inf K \leqq$ $\leqq \sup (A \cup L)$. On the other hand, $K \$ a$, so by the choice of $a, K \$ A$, i.e. $K \neq A \cup L$. Now, $A \cup B \cup C$ is normal, so inf $K$ sup $(A \cup L)$, which gives the contradiction.

II) $\inf B \leqq \inf K \cap-\sup L$. Then inf $B \leqq a$, which contradicts the choice of $a$.

This completes the proof that $A \cup B \cup C \cup\{a\}$ is normal, so the proof of the lemma is concluded.

Proof of Theorem 1. Observe that the relation

$P(x) \Leftrightarrow x$ is a code of a finite set

is recursive. It is easy to see that the relation

$T(x) \Leftrightarrow x$ is a code of a normal set of elements of $\mathscr{A}$

is recursive to $\mathscr{A}$. Define a function $g$ as follows:

$$
g(x, y, z)=\left\{\begin{array}{l}
\mu a(a \text { satisfies the claim of Lemma } 2) \text { if } x, y \text { and } z \text { are respec- } \\
\text { tively codes of the sets } A, B \text { and } C \text { satisfying the con- } \\
\text { ditions of Lemma } 2, \\
0 \quad \text { otherwise }
\end{array}\right.
$$

Then $g$ is a total function recursive in $\mathscr{A}$. Fld $(X)$ is recursive in a set $X$, so for some total injective function $a(x)$, which is recursive in $X$,

Fld $(X)=\{a(0), a(1), \ldots\}$.

For any total function $h(x)$ and $n \geqq 0$, let

$$
\begin{aligned}
& A(h, n)=\left\{h(k): a(k)<_{X} a(n+1), k \leqq n\right\}, \\
& B(h, n)=\left\{h(k): a(n+1)<_{X} a(k), k \leqq n\right\}, \\
& C(h, n)=\left\{h(k): a(k) \|_{X} a(n+1), k \leqq n\right\} .
\end{aligned}
$$

Let $b$ be an arbitrary element of $\mathscr{A}$ such that $0<b<1$. Define a function $h$ as follows:

$$
\begin{aligned}
& h(0)=b \\
& h(n+1)=g(\langle A(h, n)\rangle,\langle B(h, n)\rangle,\langle C(h, n)\rangle) .
\end{aligned}
$$

$h$ is a well defined total function. It is easy to see that $h$ is recursive in $X, \mathscr{A}$. Finally define

$$
f(a(n))=h(n) \quad \text { for } n \geqq 0 .
$$

We claim that $f$ is the required function. Observe that

$$
f(x)=y \Leftrightarrow \exists n(x=a(n) \wedge y=h(n)),
$$


so $f$ is recursive in $X, \mathscr{A}$. By induction on $k$, we prove that for all $k$,

I) $a(i)<_{x} a(j)$ if and only if $f(a(i))<f(a(j))$ for all $i, j \leqq k$, and

II) the set $\{f(a(i)): i \leqq k\}$ is normal.

Observe that the set $\{f(a(0))\}$ is normal, so I) and II) are true for $k=0$.

Suppose that I) and II) are true for $k$. Then I) implies that

$$
A(h, k)<B(h, k), C(h, k) \$ A(h, k) \text {, and } B(h, k) \neq C(h, k) \text {. }
$$

Also $A(h, k) \cup B(h, k) \cup C(h, k)=\{f(a(i)): i \leqq k\}$ so it is a normal set. Thus the sets $A=A(h, k), B=B(h, k), C=C(h, k)$ satisfy the claim of Lemma 2.

Now, $g(\langle A(h, k)\rangle,\langle B(h, k)\rangle,\langle C(h, k)\rangle)=f(a(k+1))$, so by the definition of the function $g$,

$$
\sup A(h, k)<f(a(k+1))<\inf B(h, k),
$$

$f(a(k+1)) \| C(h, k)$ and $A(h, k) \cup B(h, k) \cup C(h, k) \cup\{f((a(k+1))\}$ is a normal set. Observe now that for $i<k+1$,

$$
\begin{aligned}
& a(i)<_{X} a(k+1) \Leftrightarrow f(a(i)) \in A(h, k) \Leftrightarrow f(a(i))<f(a(k+1)) \\
& a(k+1)<_{X} a(i) \Leftrightarrow f(a(i)) \in B(h, k) \Leftrightarrow f(a(k+1))<f(a(i)) \\
& a(i)\left\|_{X} a(k+1) \Leftrightarrow f(a(i)) \in C(h, k) \Leftrightarrow f(a(i))\right\| f(a(k+1)) .
\end{aligned}
$$

Thus I) and II) are true for $k+1$. Hence by induction for all $i$ and $j$,

$$
a(i)<_{X} a(j) \Leftrightarrow f(a(i))<f(a(j)) .
$$

Since $f$ is also injective it is an embedding of $X$ into $\mathscr{A}$.

It remains to show that $f$ destroys all suprema and infima. Suppose that for some $i, j, k, a(i) \|_{X} a(j)$ and $a(i) \cup a(j)=a(k)$. Then $a(i)<_{X} a(k)$ and $a(j)<_{X} a(k)$, so $f(a(i))<f(a(k))$ and $f(a(j))<f(a(k))$. The set $\{f(a(n)): n$ $\leqq \max (i, j, k)\}$ is normal, thus

$$
f(a(i)) \cup f(a(j))<f(a(k)),
$$

i.e. $f$ destroys the supremum $a(i) \cup a(j)$. The same argument applies in the case of infinum of $X$-incomparable elements. This concludes the proof of the theorem.

\section{Embedding preserving suprema and infima. Let}

$$
A=\left\{x: \operatorname{Seq}(x) \wedge \forall i\left(i<\operatorname{lh}(x) \Rightarrow\left((x)_{i}=0 \vee(x)_{2}=1\right)\right)\right\} .
$$

Thus $A$ is the set of codes of all finite sequences of zeroes and ones.

Let $\cup$ and $\cap$ be some operations on $A$ satisfying the following property:

$$
\text { If }\left\langle k_{1}, \ldots, k_{n}\right\rangle \in A \text {, }
$$


then

$$
\begin{aligned}
\left\langle k_{1}, \ldots, k_{n}, 0\right\rangle \cup\left\langle k_{1}, \ldots, k_{n}, 1\right\rangle & =\left\langle k_{1}, \ldots, k_{n}\right\rangle \\
\left\langle k_{1}, \ldots, k_{n}, 0\right\rangle \cap\left\langle k_{1}, \ldots, k_{n}, 1\right\rangle & =\langle 0\rangle .
\end{aligned}
$$

Let $\mathscr{M}$ be the Boolean algebra generated by $\mathscr{A}$ and by operations $\cup$ and $\cap$ satisfying the above property. It is well known that $\mathscr{M}$ is (isomorphic to) the Boolean algebra of all clopen subsets of the Cantor Space. The elements of $\mathscr{M}$ are just all the finite joins and meets of $\mathscr{A}$.

It is easy to see that $\mathscr{M}$ is recursive, that is to say

$$
\mathscr{M}=\left\langle A_{\mathscr{M}}, \cup, \cap,-, 0,1\right\rangle,
$$

where $A_{\mathscr{M}}$ is a recursive set and the graphs of partial functions $\cup, \cap$ and - are recursive. $\mathscr{M}$ is an atomless Boolean algebra.

We prove the following theorem.

THEOREM 2. There exists a recursive partial ordering $X$ on $\omega$, such that

I) there is an embedding of $X$ into $\mathscr{M}$ which preserves all suprema and infima of $X$, and

II) no such embeddings are recursive.

Proof. Let $P(x)$ be a $\Sigma_{2}{ }^{0}-\Pi_{2}{ }^{0}$ relation. For some recursive $R$, $P(x) \Leftrightarrow \exists y \forall z R(x, y, z)$.

Define a partial function $g$ as follows:

$$
g(x, y) \simeq\langle x, y, \mu z\urcorner R(x, y, z)\rangle
$$

Observe that graph $(g)$ is recursive. Define

$$
h(x, y) \simeq\langle g(x, 0), \ldots, g(x, y-1)\rangle \text { where } y-1=\max (y-1,0) .
$$

Clearly $h$ is a partial recursive function. Observe that

1) $(h(x, y)$ is defined and $z<y) \Rightarrow(h(x, z)$ is defined $)$

2) For all $x[\lambda y h(x, y)$ is total $\Leftrightarrow \lambda y g(x, y)$ is total]

3) $\operatorname{graph}(h)(x, y, z) \Leftrightarrow \operatorname{Seq}(z) \wedge \mathrm{lh}_{(z)}=y \wedge \forall i(i<y \Rightarrow \operatorname{graph}(g)$ $\left(x, i,(z)_{i}\right)$. Our ordering $X$ looks as follows:

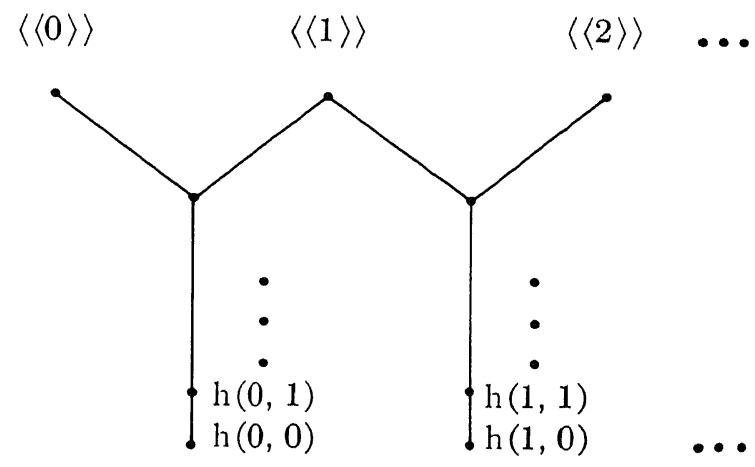


More formally,

$$
\begin{aligned}
& X=\{(\langle\langle x\rangle\rangle,\langle\langle x\rangle\rangle): x \geqq 0\} \cup\{(h(x, m), h(x, n)): x \geqq 0, n \geqq m \geqq 0\} \\
& \cup\{(h(x, m),\langle\langle x\rangle\rangle): m \geqq 0, x \geqq 0\} \\
& \cup\{(h(x, m),\langle\langle x+1\rangle\rangle): x \geqq 0, m \geqq 0\rangle\} .
\end{aligned}
$$

$X$ is clearly a recursive set. Now let $T$ be the following relation:

$$
T(x) \Leftrightarrow\langle\langle x\rangle\rangle \cap\langle\langle x+1\rangle\rangle \text { exists. }
$$

Then

$$
\begin{aligned}
T(x) & \Leftrightarrow \lambda y h(x, y) \text { is not total, } \\
& \Leftrightarrow \lambda y g(x, y) \text { is not total, } \\
& \Leftrightarrow \exists y(g(x, y) \text { is not defined }), \\
& \Leftrightarrow \exists y(\forall z R(x, y, z)), \\
& \Leftrightarrow P(x) .
\end{aligned}
$$

Hence $T$ is a $\Sigma_{2}{ }^{0}-\Pi_{2}{ }^{0}$ relation.

It is easy to see that there is an embedding of $X$ into $\mathscr{M}$ which preserves all suprema and infima of $X$. Let $f$ be such an embedding. Then

$$
T(x) \Leftrightarrow \exists z(z \in \operatorname{Fld}(X) \wedge(f(\langle\langle x\rangle\rangle) \cap f(\langle\langle x+1\rangle\rangle)=f(z)) .
$$

Thus, if $f$ was recursive then $T$ would be a $\Sigma_{1}{ }^{0}$ set, which is not the case. Hence no such embeddings are recursive, completing the proof.

The above theorem shows that Theorem 1 is not true when $I$ ) is changed for

$\left.I^{\prime}\right) f$ preserves all suprema and infima of $X$.

We pass now to the problem of recursive embeddings of Boolean algebras into Boolean algebras. Abian in [1] proves the following lemma.

Lemma 3. (Abian). Let $\mathscr{A}$ and $\mathscr{B}$ be countable Boolean algebras and let $\mathscr{B}$ be atomless. Let $f$ be an isomorphism from a finite subalgebra $\mathscr{A}_{1}$ of $\mathscr{A}$ onto a finite subalgebra $\mathscr{B}_{1}$ of $\mathscr{B}$. Then for every a $\in \mathscr{A}-\mathscr{A}_{1}$ there exists $b \in \mathscr{B}-\mathscr{B}_{1}$ such that the assignment $f(a)=b$ extends the isomorphism $f$ from the subalgebra of $\mathscr{A}$ generated by $\mathscr{A}_{1} \cup\{a\}$ onto the subalgebra of $\mathscr{B}$ generated by $\mathscr{B}_{1} \cup\{b\}$.

Using this lemma, Abian gives an algebraic proof of the well-known theorem that two countable atomless Boolean algebras are isomorphic. In fact this isomorphism is recursive in the considered algebras. More precisely, we have the following theorem.

Theorem 3. Let $\mathscr{A}$ and $\mathscr{B}$ be countable Boolean algebras on $\omega$ and let $\mathscr{B}$ be atomless. Then

I) there exists an embedding of $\mathscr{A}$ into $\mathscr{B}$ (as Boolean algebras) which is recursive in $\mathscr{A}, \mathscr{B}$, and 
II) if $\mathscr{A}$ is atomless, then there exists an isomorphism of $\mathscr{A}$ and $\mathscr{B}$ which is recursive in $\mathscr{A}, \mathscr{B}$.

Proof. I) follows by the repeated use of Lemma 3. II) follows using Lemma 3 repeatedly back and forth. It is clear that in both cases the constructed embedding $f$ is recursive in $\mathscr{A}, \mathscr{B}$.

Remark. This paper is closely related with the Van Emde Boas [2] paper. Van Emde Boas proves there that every recursive partial ordering can be recursively embedded into the Boolean algebra $\mathscr{M}$ defined earlier. We obtained Theorem 1 independently of his paper.

\section{REFERENCES}

1. A. Abian, Categoricity of denumerable atomless Boolean rings, Studia Logica (1972), 63-68. 2. P. van Emde Boas, Mostowski's universal set-algebra, Mathematisch Centrum report, ZII 14/73, Amsterdam, 1973.

3. J. R. Shoenfield, Mathematical logi (Addison, Mesley, Reading, Massachusetts, 1967).

4. R. Sikorski, Boolean algebras, third edition (Berlin, 1969).

Mathematical Centre, 2e Boerhaavestraat 49 , Amsterdam 1005, The Netherlands 